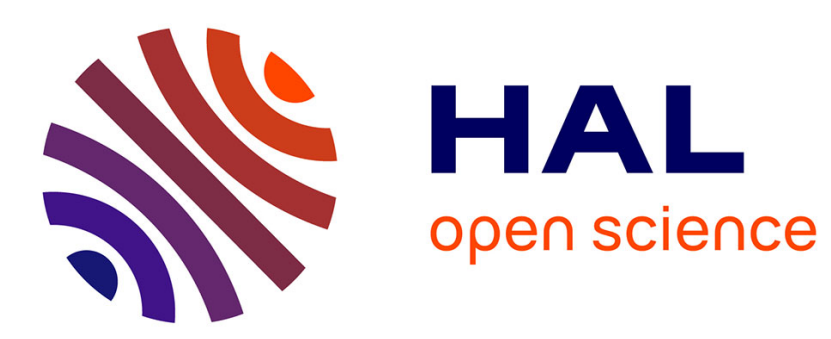

\title{
Introduction. Innovations sociales et développement des territoires dans les campagnes européennes
}

\author{
Guillaume Lacquement, Christophe Queva
}

\section{To cite this version:}

Guillaume Lacquement, Christophe Queva. Introduction. Innovations sociales et développement des territoires dans les campagnes européennes. Norois, 2016, 241, p. 7-13. 10.4000/norois.5978 . halshs01708848

\section{HAL Id: halshs-01708848 \\ https://shs.hal.science/halshs-01708848}

Submitted on 14 Feb 2018

HAL is a multi-disciplinary open access archive for the deposit and dissemination of scientific research documents, whether they are published or not. The documents may come from teaching and research institutions in France or abroad, or from public or private research centers.
L'archive ouverte pluridisciplinaire HAL, est destinée au dépôt et à la diffusion de documents scientifiques de niveau recherche, publiés ou non, émanant des établissements d'enseignement et de recherche français ou étrangers, des laboratoires publics ou privés. 


\section{Norois}

Environnement, aménagement, société

$241 \mid 2016$

Innovation sociale et développement des territoires dans les campagnes européennes

\section{Introduction. Innovations sociales et développement des territoires dans les campagnes européennes}

Social Innovations and Territorial Development in the European Rural Areas

\section{Guillaume Lacquement et Christophe Quéva}

\section{Q revues.org}

Édition électronique

URL : http://norois.revues.org/5978

DOI : $10.4000 /$ norois. 5978

ISBN : 978-2-7535-5504-4

ISSN : $1760-8546$

\section{Éditeur}

Presses universitaires de Rennes

Édition imprimée

Date de publication : 30 décembre 2016

Pagination : 7-13

ISBN : 978-2-7535-5483-2

ISSN : 0029-182X

Distribution électronique Cairn

CAIRN

CHERCHER, REPÉRER, AVANCER.

\section{Référence électronique}

Guillaume Lacquement et Christophe Quéva, «Introduction. Innovations sociales et développement des territoires dans les campagnes européennes », Norois [En ligne], 241 | 2016, mis en ligne le 31 décembre 2018, consulté le 29 mars 2017. URL : http://norois.revues.org/5978; DOI : 10.4000/ norois. 5978 


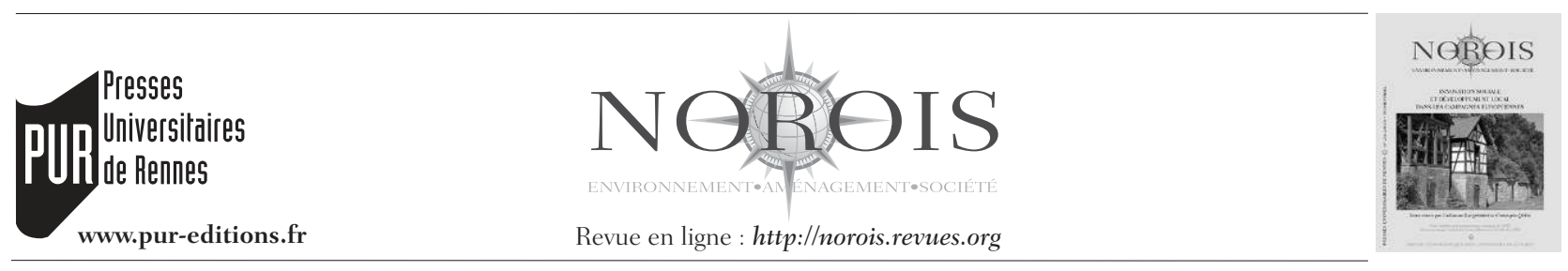

\title{
Introduction \\ Innovation sociale et développement des territoires dans les campagnes européennes
}

\author{
Social Innovations and Territorial Development in the European Rural Areas
}

Guillaume Lacquement ${ }^{a}$ et Christophe Quévab

a Professeur de Géographie, Université de Perpignan, UMR CNRS 5281 ART-Dev. (lacqueme@univ-perp.fr)
b Maître de conférences en Géographie, Université Paris 1 Panthéon-Sorbonne,UMR Géographie-Cités 8504.
(christophe.queva@univ-paris1.fr)

Ce dossier thématique s'appuie sur la valorisation d'une sélection de communications issues du colloque international de géographie rurale, « Les campagnes: espaces d'innovation dans un monde urbain », organisé en juin 2014 par l'UMR CNRS 6590 Espaces et sociétés (ESO), la Commission de Géographie rurale (CNFG), le Grupe de geografia rural, le Rural geography research group, et les Arbeitskreise Ländlicher Raum und Dorfentwicklung. Ce colloque visait à interroger la place et le rôle des espaces ruraux dans les processus de compétitivité territoriale et d'innovation, dans le contexte de sociétés de plus en plus urbaines. Dans ce cadre, les innovations analysées - envisagées soit comme l'adaptation à une contrainte, soit comme la mise à profit d'une opportunité nouvelle - concernaient à la fois les pratiques des populations rurales, les usages et les fonctions des campagnes, ou encore les modalités d'organisation, d'aménagement et de développement des territoires ruraux. C'est dans cette dernière perspective que s'inscrit ce dossier thématique, dont l'enjeu est d'analyser la diffusion des outils et des démarches de développement territorial dans plusieurs régions rurales de l'Europe communautaire. Il se concentre sur les formes, les modalités et la diversité des processus d'innovation sociale pour interroger la manière dont se combinent l'introduction d'une nouveauté institutionnelle et la capacité organisationnelle des sociétés locales à l'appliquer ou à la réinventer. À cette fin, le dossier met la focale sur le programme européen LEADER (Liaisons Entre Actions de Développement de l'Économie Rurale), en tant que démarche de mobilisation des acteurs locaux et dispositif de création de ressources en faveur du développement et de l'attractivité des territoires ruraux.

\section{LES INNOVATIONS SOCIALES ENTRE RÉSEAUX D'ACTEURS, RESSOURCES SOCIALES ET CAPITAL SOCIAL}

La notion d'innovation sociale «se définit par son caractère novateur ou hors normes et par l'objectif général qu'elle poursuit, soit celui de favoriser le mieux-être des individus et des collectivités. Elle se caractérise tout autant par un processus de mise en œuvre impliquant une coopération entre une diversité d'acteurs que par les résultats obte- 
nus, immatériels ou tangibles »(Cloutier, 2003). Dans le cadre plus spécifique du développement local et des projets de territoires, "l'innovation sociale correspond aux changements au niveau tant institutionnel que des comportements collectifs et individuels (personnes éminentes, leadership) contribuant à l'intégration sociale » et à un développement territorial intégré (Hillier et al., 2004). Ces dynamiques de coordination de l'action collective peuvent être considérées comme un facteur de développement des territoires, contribuant à structurer localement des réseaux d'acteurs porteurs de projets. L'innovation ne se réduit pas à l'introduction de techniques nouvelles de production ou de service dans les activités économiques. Elle comprend une dimension sociale qui s'exprime dans un processus d'interactions entre des individus en situation d'apprentissage, favorisant la création d'activités nouvelles ou reconfigurées (Dargan, Shucksmith, 2008).

Ce système d'innovation se structure en réseaux, au sein desquels les acteurs sociaux partagent des représentations, se distribuent des responsabilités ou des rôles, édictent des règles ou des normes, permettant de faire circuler l'information, de distribuer les ressources et de prendre des décisions. Pour le courant de l'économie territoriale, les réseaux se forment à partir de logiques de proximité (Torre, Filippi, 2005). Cela concerne non seulement les distances géographiques qui en séparent ou en rapprochent les membres, mais aussi les liens qui s'établissent entre eux, en fonction de leur appartenance sociale et des valeurs qu'ils partagent (Bouba-Olga, Carrincazeau, Coris, 2008; Torre, 2009). Ils se constituent en institutions formelles ou informelles qui coordonnent l'action collective en faveur du développement territorial. L'institutionnalisation se lit ainsi à travers la création de structures nouvelles de coordination et d'impulsion, de partenariats divers, comme les groupes d'action locale (GAL) des programmes européens LEADER.

L'action collective en faveur du développement économique dépend alors des relations interpersonnelles qui se développent au sein de ces réseaux. La sociologie structurale postule que les phénomènes économiques sont, de cette manière, "encastrés » (embedded) dans les relations sociales (Granovetter, 1985). La décision de financer un projet de territoire ou une action de développement résulte d'un processus de négociation, pris dans un jeu de relations interpersonnelles (Rambonilaza, 2010). Les liens établis et les interactions entre les individus tissent une matrice de ressources sociales qui accompagne la réalisation des projets (Burt, 1992). Cette matrice de relations plus ou moins denses, plus ou moins équivalentes, mais dans tous les cas interdépendantes (Forsé, 2008), constitue un capital social qui crée une sorte de valeur ajoutée (Lin, 1995) et qui détermine la capacité d'action des structures partenariales à finaliser des projets et en entreprendre de nouveaux.

\section{LE DÉVELOPPEMENT DES TERRITOIRES AU PRISME DE L'INNOVATION SOCIALE}

Construit social, l'innovation revêt également une dimension territoriale. La réflexion à ce sujet a été initiée par Philippe Aydalot en lien étroit avec celle du développement endogène (ou développement local), considéré comme le résultat abouti d'une société locale innovatrice (Aydalot, 1985). Les interactions sociales qui animent les institutions partenariales se doublent d'interactions territoriales, dans le sens où elles s'inscrivent dans un ensemble territorialisé, régi par des normes, des règles, des valeurs qui sont autant de mécanismes agissant sur le comportement des individus et sur les relations qu'ils entretiennent (Fontan et al., 2004 ; Camagni, Maillat, 2006; Crevoisier, 2006). Elles forment un milieu innovateur qui se distingue par sa capacité à formuler des projets, à identifier et à valoriser des ressources économiques nouvelles conduisant à l'adaptation et au renouvellement des systèmes de production (Kébir, Maillat, 2004).

L'interprétation de l'inscription territoriale de l'innovation sociale conduit à considérer l'existence d'un capital territorial (Camagni, 2006). Il se présente sous la forme d'une matrice qui exprime la capacité des sociétés locales à traduire la proximité géographique et relationnelle en réseau de coopération partenariale, et se saisir des éléments matériels et immatériels du territoire local pour concevoir et valoriser des ressources économiques nouvelles (Camagni, 2013). Envisagé comme construction sociale et territoriale, le processus d'innovation est sensible aux effets de contextes géographiques qui différencient les formes de coordination et de mise en réseau des acteurs impliqués dans le dévelop- 
pement économique et la gestion des territoires (Fontan et al., 2004).

\section{LES CAMPAGNES, ANGLES-MORTS OU FOYERS D'ÉMERGENCE POUR LES INNOVATIONS?}

Au premier abord, les espaces ruraux constituent un terreau a priori peu propice à l'affirmation de logiques innovantes : la faible densité qui les caractérise s'associe à un "agencement sociospatial singulier»(Barthe, Milian, 2011), marqué par les caractères suivants : " occupation humaine distendue et faible empreinte des infrastructures, activités principales basées sur la valorisation agricole et forestière, importance des espaces ouverts et rapports privilégiés à la nature » (ibid.). C'est en ce sens que les campagnes ont longtemps été, en France, comme dans les autres pays européens, associées à l'idée de crise rurale, constituant de véritables "synecdoques de la déprise rurale et agricole »(Clarimont et al., 2006). Or, depuis les années 1990, à la suite des travaux de B. Kayser (1990) sur la « renaissance rurale » dans les pays industrialisés, les études statistiques et scientifiques sur les espaces ruraux montrent que les campagnes - même les plus isolées - connaissent des mutations positives : croissance démographique avec notamment l'installation de "néoruraux », développement de nouvelles fonctions (environnementale, touristique, résidentielle, de loisirs, etc.) dans l'espace rural, affirmation de nouvelles représentations spatiales, fondées notamment sur un idéal de quiétude, un besoin d'espace et d'accès à la nature, etc. Ce changement de regard et de posture vis-àvis des campagnes des pays industrialisés s'associe également à l'identification de potentialités ou de ressources rurales à valoriser : «La faible densité, d'abord interprétée sur le mode négatif, assure la cohésion d'un groupe à la recherche de solutions [...]. Elle n'est plus exclusivement définie en termes de problème, elle devient parfois un atout à valoriser, un élément fondateur de l'attractivité nouvelle de ces territoires ruraux » (Clarimont et al., 2006).

Dans cette perspective, les enjeux actuels d'aménagement des espaces ruraux relèvent moins d'une logique d'assistance par les politiques publiques (pour des campagnes identifiées avant tout comme des espaces de contraintes et de handicaps à revi- taliser) que d'une logique de projets de développement local et d'innovations, portée par les acteurs locaux (Quéva, Brès, 2016). Caractérisées par le « déploiement d'une économie diffuse peu polarisée mais structurante en matière de tissu d'activités et d'emplois, et donc de maintien d'une population permanente » (Barthe, Milian, 2011), les campagnes peu densément peuplées sont moins marquées par les rapports hiérarchiques ou de domination que dans le cas d'espaces concentrés ou polarisés, où un centre, un pôle urbain, exerce une influence sur son espace environnant (Quéva, 2015). Dès lors, le développement local dans les espaces ruraux est envisagé comme une dynamique endogène, initiée par des acteurs locaux porteurs de projets, qui peut se voir accompagnée et renforcée par des politiques publiques, nationales ou européennes.

\section{LE PROGRAMME EUROPÉEN LEADER, UN ACCÉLÉRATEUR D'INNOVATIONS DANS LES TERRITOIRES RURAUX?}

Le programme européen LEADER fait partie des politiques publiques qui encouragent l'émergence de systèmes d'action locaux destinés à promouvoir les initiatives endogènes de développement et d'intégration socio-économique des territoires ruraux. Il introduit une forme nouvelle de gouvernance des territoires, en invitant les acteurs locaux à prospecter et à valoriser des ressources alternatives, afin de diversifier les activités rurales. L'innovation se situe donc dans la genèse d'une action politique de niveau local mettant en scène des acteurs représentatifs de la société rurale et les impliquant dans un ensemble d'arrangements institutionnels. La composition et le fonctionnement des réseaux de coopération du programme LEADER (les groupes d'action locale ou GAL) déterminent alors le pilotage des initiatives endogènes et le contenu des projets de développement. La diffusion de cette innovation sociale en Europe s'opère de manière différenciée en raison de la diversité des trajectoires territoriales et des effets de contexte géographique (Maurel, Chevalier, Lacquement, 2014).

Dans quelle mesure l'émergence et la diffusion des innovations sociales dans les espaces ruraux relèventelles avant tout d'initiatives locales ou plutôt de stratégies d'accompagnement par les politiques publiques - de l'échelle européenne aux échelles nationales 
et régionales? Plus spécifiquement, en quoi les démarches soutenues par le programme européen LEADER relèvent-elles de logiques innovantes sur les plans social et territorial?

Nous formulons trois hypothèses principales pour appréhender ces questionnements. La première est que, dans le contexte des pays industrialisés et notamment européens, les espaces ruraux sont potentiellement porteurs d'innovations sociales, les acteurs locaux contribuant de plus en plus à élaborer des projets tournés vers la valorisation des potentialités de ces territoires. Cette perspective s'inscrit dans la continuité des travaux récents sur les dynamiques des espaces de faible densité, identifiant paradoxalement les fragilités des espaces ruraux comme les possibles déclencheurs d'une réactivité forte de la part des populations et acteurs locaux : "l'inventivité, dans un contexte de fortes contraintes (économiques, politiques) traverse les pratiques sociales renforçant l'hypothèse que ces espaces pourraient être des laboratoires d'expérimentation dans différents registres à disposition de l'ensemble de la société »(Barthe, Milian, 2011).

Notre deuxième hypothèse porte sur la distinction nécessaire entre l'émergence de l'innovation dans les espaces ruraux d'une part, et la diffusion de l'innovation d'autre part. Nous considérons ainsi que, pour la première, la place des encadrements - par les politiques publiques des États européens et de l'Union européenne - reste potentiellement importante en tant que déclencheurs des projets innovants, alors que la diffusion de l'innovation se fonderait principalement sur des enjeux locaux et de proximité, par le rôle de l'ingénierie et des réseaux locaux de coopération.

Enfin, notre troisième hypothèse considère que les logiques d'innovation sociale à l'œuvre dans les territoires ruraux contribuent également à structurer et à faire émerger des constructions territoriales fortes - autour des territoires LEADER et des territoires de développement local en général - marquées par des logiques fonctionnelles et identitaires de plus en plus marquées.

\section{AU CROISEMENT DES DISCIPLINES ET DES MÉTHODES}

Dans le dossier qui est ici proposé, l'analyse de la diffusion des outils et des démarches de déve- loppement territorial dans les pays européens, tout en conservant une perspective géographique, croise des approches et des outils issus de différentes disciplines. Les contributions de ce dossier font référence à l'appareil conceptuel élaboré par les études de transfert de politiques publiques (Delpeuch, 2008, 2009) (policy tranfer studies) (Wolman, Page, 2002) et empruntent les méthodes de l'économie spatiale (Gaudard, 2004; Coulet, Pecqueur, 2013) pour étudier les stratégies et les projets de développement portés par les acteurs locaux. Pour décrire le mode de fonctionnement des systèmes d'action locaux, les analyses mobilisent les outils conceptuels et méthodologiques de la sociologie politique (Muller, 2000), comme par exemple l'analyse des réseaux (Mercklé, 2011). L'efficience économique de l'innovation sociale est évaluée au prisme de la notion de «coût de transaction » (Coase, 2005; Williamson, 1994). En outre, la pluralité des terrains étudiés (France, Irlande, Italie, Allemagne et Hongrie) inscrit les analyses dans un cadre international et comparatif. La confrontation des terrains d'enquête permet, au-delà de la démarche monographique, d'explorer le processus de diffusion de l'innovation au regard de régimes territoriaux et de contextes géographiques différents.

Trois enseignements principaux peuvent être retenus de ces croisements disciplinaires. Tout d'abord, les quatre études considèrent l'innovation sociale au sein du programme européen LEADER comme l'apprentissage des règles et des normes de la gouvernance locale. Ces dernières construisent un modèle de gestion des territoires qui se situe à l'articulation de logiques endogènes et exogènes, les initiatives des acteurs locaux étant contractualisées par des dispositifs établis par l'emboîtement des échelons du système territorial et par l'application du principe de subsidiarité (Union européenne, États nationaux, régions). Cette approche institutionnelle s'oppose à la vision normative de l'innovation qui se focalise sur la fabrication de nouveaux produits ou sur l'utilisation de nouvelles techniques de production ou de services. Elle met également en cause la conception classique de l'administration et de la gestion des territoires, la manière de penser les politiques publiques. Elle suppose que les institutions de gouvernement n'ont plus le monopole de l'action publique, qui se trouve déléguée à des acteurs de statuts divers, organisés en réseaux pour concevoir 
des projets de territoire et coordonner une action collective de développement.

Le deuxième enseignement concerne les conditions de cet apprentissage. La démarche LEADER, décrite comme une démarche bottom up ou ascendante, suppose trois conditions principales. La première renvoie à l'application d'un processus de décentralisation, qui prévoit la délégation de compétences de gestion territoriale au niveau local, et qui a pour conséquence de faire émerger de nouvelles relations entre les différents niveaux du système territorial (supranational, national, régional et local). Dans le cadre de l'application du programme européen, il s'agit de relations de contractualisation. Mais le transfert de prérogatives crée des situations de conflit et contraint à la négociation en ce qui concerne la répartition des tâches. La deuxième condition tient à la création de partenariats tripartites, dont la structuration et le fonctionnement obéissent à des règles nouvelles et déterminent le capital social des territoires locaux. La démarche LEADER suppose la mobilisation, au sein des groupes d'action locale (GAL), d'individus issus à la fois de la sphère publique, du monde de l'entreprise et du secteur associatif. Les configurations d'acteurs dans les GAL se composent alors par des jeux complexes de négociation et d'interprétation des équilibres de la représentativité. La démarche LEADER s'exprime en troisième lieu dans la coordination d'une action collective qui implique la délimitation d'un périmètre d'application, la conception d'une stratégie de développement et la sélection de projets de valorisation des ressources locales.

Cette manière de territorialiser les politiques publiques de développement économique présente néanmoins des limites. Le troisième enseignement de ce dossier pointe le fait que l'apprentissage de la démarche LEADER dépend de structures d'ingénierie territoriale qui facilitent la traduction opérationnelle de la politique d'intervention. Les groupes d'action locale sont portés par des structures territoriales organisées en syndicats mixtes (comme les parcs naturels régionaux) ou en association (comme les Pays). L'équipe d'animation de ces structures joue un rôle décisif dans la préparation du dossier de candidature, dans la construction des équilibres de la composition des GAL, dans la rédaction des documents programmatiques, dans l'instruction et la programmation des projets, ainsi que dans la ges- tion des enveloppes budgétaires. L'ingénierie participe de l'efficience des politiques publiques, mais encadre au final au moins partiellement l'apprentissage de la gouvernance locale.

\section{LE POIDS DES CONTEXTES TERRITORIAUX}

Les quatre contributions, prenant appui sur une pluralité de terrains en France et en Europe, montrent que le dispositif LEADER s'est progressivement imposé comme l'un des lieux privilégiés d'innovation en matière d'apprentissage des principes du développement endogène et de la gouvernance locale. Le transfert des politiques de développement rural au sein des pays de l'Union européenne s'illustre par une certaine convergence des pratiques de gestion du développement socioéconomique dans les zones rurales. Cependant, les études localisées montrent également que l'apprentissage de la démarche LEADER est sensible aux effets de contexte géographique. Cela concerne le cadre institutionnel des États nationaux, qui régit le processus de décentralisation et de redistribution des prérogatives de gestion territoriale, ainsi que les modalités d'application du programme européen. Cela concerne tout autant le cadre spatial, c'està-dire les propriétés spécifiques des lieux et les formes de l'organisation de l'espace, qui influent sur les jeux sociaux et les stratégies développées par les acteurs locaux. La démarche LEADER donne lieu à un apprentissage spatialement différencié. En tant qu'innovation sociale, elle s'inscrit dans un processus territorialisé. C'est ce que nous enseigne la lecture croisée de ces études. Le dossier part du contexte français (M. Berriet-Solliec, D. Lépicier et D. Vollet), et élargit progressivement les perspectives à l'échelle européenne - du contexte de l'Europe occidentale (M. Cawley) à celui de l'Europe orientale (P. Chevalier et G. Lacquement) en passant par le cadre italien (M. Labianca).

Le premier article de Marielle Berriet-Solliec, Denis Lépicier et Dominique Vollet prend le parti de questionner l'efficience de la politique publique en évaluant ses coûts de mise en œuvre. L'approche économique s'intéresse aux coûts de transaction du programme LEADER - c'est-à-dire aux coûts de gestion, d'animation et de suivi de son application - dans deux régions françaises, l'Auvergne et 
la Bourgogne, retenues à partir de critères morphofonctionnels (la place des espaces ruraux dans le développement régional) et gestionnaires (modes passés et présents de gestion des politiques territoriales). L'étude propose une estimation du niveau et de la variabilité des coûts propres au programme LEADER pour le mettre en regard avec le bénéfice des actions menées. L'analyse mesure ainsi le « prix à payer » pour innover et renouveler l'action publique en faveur du développement des espaces ruraux. Elle souligne l'impact des coûts de transaction sur la démarche LEADER, c'est-à-dire sur le mode de coordination de l'action locale, qui tendent à favoriser les projets portés par les collectivités territoriales aux dépens de ceux portés par des opérateurs privés. Elle conclut alors aux enjeux politiques et financiers que représente l'ingénierie territoriale dans l'application des politiques publiques ascendantes et contractualisées.

La contribution de Mary Cawley sur le programme LEADER en Irlande se rapporte à un autre enjeu tout aussi important, l'enjeu politique et institutionnel de la redistribution des compétences de gestion territoriale. L'auteure montre comment l'application des principes de la gouvernance locale a mis en cause les structures du gouvernement local. L'introduction du programme européen au début des années 1990 a bénéficié dans ce pays de la tradition associative qui compensait le déficit d'intervention des collectivités locales en matière de gestion des services ruraux. Les structures associatives se sont largement impliquées dans la création et l'animation des groupes d'action locale (GAL) institués par le programme LEADER. Mais l'extension progressive des GAL a créé un nouveau maillage d'intervention qui est venu transgresser à l'échelle locale le maillage administratif des comtés. La délégation de prérogatives de gestion des territoires ruraux aux nouvelles structures partenariales est venue émousser les fonctions des administrations locales alors même qu'une réforme de l'État transformait leur fonctionnement selon les principes néolibéraux du new public management. L'étude suit l'évolution de la situation de tension et de conflit qui s'est alors établie entre les formes nouvelles de démocratie participative et les structures réformées de démocratie représentative. La description des réformes administratives successives et des dispositifs de coordination des politiques rurales permet de comprendre le rôle d'arbitre conservé par l'État central, qui au nom de l'efficience des politiques publiques, accroît les compétences des collectivités locales dans le pilotage et la coordination des programmes de développement rural, revenant au final à leur attribuer la responsabilité du programme LEADER.

L'article de Marinela Labianca apporte un éclairage complémentaire intéressant dans le contexte d'une région rurale italienne, les Pouilles, marquée par la fragilité économique et relevant du zonage «Convergence » de l'Union européenne. En analysant les documents d'aménagement régional pour le développement rural (2007-2013), ainsi que les textes des appels à projets entre 2000-2015, l'auteure propose un regard critique sur les modèles de gouvernance qui se dessinent. Si elle note l'importance et la nécessité des innovations sociales dans le contexte des espaces ruraux fragilisés, l'étude relève également un certain nombre de difficultés de mise en œuvre. À travers une analyse textuelle, Marinela Labianca pointe ainsi l'influence de l'Union européenne et de l'État italien dans la formulation des objectifs régionaux et locaux, ainsi que la mobilisation d'un champ lexical de l'innovation largement influencé par un cadre européen prescriptif, limitant l'autonomie des acteurs et des territoires locaux. En termes de contenus de projets, l'auteure note que l'innovation sociale reste très secondaire par rapport à l'innovation économique centrée sur les systèmes productifs et la compétitivité des entreprises. La rhétorique dominante du développement rural dans les Pouilles repose ainsi avant tout sur une approche productiviste, dans le cadre d'un modèle de gouvernement prescriptif, où les processus participatifs ne jouent qu'un rôle globalement secondaire. Au-delà de cette tendance générale, certains GAL n'en restent pas moins innovants sur le plan social, en proposant des projets de gouvernance réinventés, notamment dans le cadre de réseaux d'acteurs locaux marqués par une assise territoriale forte.

C'est également ce poids des effets de contextes qui est mis en avant dans l'article de Pascal Chevalier et Guillaume Lacquement, centré sur l'analyse du programme LEADER dans deux régions marquées par l'héritage socialiste et la transition à l'économie de marché : le Land de Thuringe en Allemagne (ex-RDA), et le comitat de Baranya en Hongrie. L'innovation sociale y est analysée au 
prisme de deux groupes d'action locale (GAL), dont les auteurs analysent les modalités, les forces et les fragilités, dans le contexte d'une décentralisation et d'une autonomisation des collectivités territoriales en rupture systémique avec l'héritage communiste. Après avoir présenté le contexte et les modalités de mise en œuvre du programme dans les deux États, P. Chevalier et G. Lacquement analysent de façon fine le fonctionnement des GAL en recourant aux méthodes de la sociologie structurale, permettant de donner à voir la nature, l'intensité et les déséquilibres des relations au sein des réseaux de gouvernance constituant les GAL. Les auteurs mettent ainsi en évidence des liens d'interconnaissance, permettant de mesurer les degrés d'intégration sociale et de faire ressortir une structuration d'ensemble des deux GAL étudiés, plus ou moins équilibrée. Les leaderships identifiés et resitués dans leurs temporalités par les deux auteurs permettent de différencier des logiques de gouvernance plus ou moins ancrées territorialement, intégratrices ou excluantes, le poids des contextes nationaux et des spécificités locales jouant un rôle central dans la compréhension de ces différenciations.

\section{Bibliographie}

Barthe L., Milian J., 2011. Les espaces de la faible densité, processus et scénarios, Territoires 2040, n 4, p. 151-183.

Burt R.S., 1992. Structural holes: the social structure of competition, Harvard University Press, Cambridge, MA, 324 p.

Bouba-Olga O., Carrincazeau C., Coris M., 2008. La proximité : 15 ans déjà, Revue d'Économie Régionale et Urbaine, 3, p. 279-287.

Clarimont, S., Aldhuy, J., Labussière O., 2006. Les recompositions territoriales face à la faible densité : comparaison des « pays » aquitains et des comarcas aragonaises, Annales de géographie, $\mathrm{n}^{\circ}$ 647, p. 26-48.

Cloutier J., 2003. Qu'est-ce que l'innovation sociale?, Cahier $d u$ CRISES, coll. «Études théoriques; ET0314 », 60 p.

Coase R., 2005. L'entreprise, le marché et le droit, Paris, Éditions d'Organisation, $243 \mathrm{p}$.

Coulet C., Pecqueur B., 2013. L'économie territoriale, 2e édition, Grenoble, Presses Universitaire de Grenoble, 144 p.

Dargan L., Shucksmith M., 2008. LEADER and Innovation, Sociologia Ruralis, vol. 38, n 3, p. 274-291.
Delpeuch T., 2008. L'analyse des transferts internationaux de politiques publiques : un état de l'art, Questions de recherche, CERI/Sciences Po, $\mathrm{n}^{\circ} 27,69 \mathrm{p}$.

Delpeuch T., 2009. Comprendre la circulation internationale des solutions d'action publique : panorama des policy transfer studies, Critique internationale, vol. 2, n 3, p. 153-165.

Hillier J. et al., 2004. Trois essais sur le rôle de l'innovation sociale dans le développement territorial, Géographie, Économie, Société, 2, vol. 6, p. 129-152.

FonTAN J.-M. et al., 2004. Innovation et société, pour élargir l'analyse des effets territoriaux de l'innovation, Géographie, Économie et Société, 2, vol. 6, p. 115-128.

Fonsé M., 2008. Définir et analyser les réseaux sociaux, les enjeux de l'analyse structurale, Informations sociales, vol. 3, $n^{\circ} 147$, p. 10-19.

Gaudard G., 2004. La nouvelle économie spatiale, Revue d'Économie Régionale et Urbaine, 3, p. 453-463.

Granovetter M., 1985. Economic action and social structure: The problem of embededdness, American Journal of Sociology, vol. 91, n³, p. 481-510.

KaYser B., 1990. La renaissance rurale. Sociologie des campagnes du monde occidental, Paris, Armand Colin, 316 p.

LiN N., 1995. Les ressources sociales : une théorie du capital social, Revue Française de Sociologie, vol. 36, n 4, 1995 , p. 685-704.

Maurel M.-C., Chevalier P., Lacquement G., 2014. Transfert et apprentissage du modèle LEADER en Europe centrale, Paris, L'Harmattan, coll. «Questionner l'Europe », 300 p.

Mercklé P., 2004. Sociologie des réseaux sociaux, Paris, La Découverte, nouvelle édition, 2011, 128 p.

Muller P., 2000. L'analyse cognitive des politiques publiques, vers une sociologie politique de l'action publique, Revue française de science politique, $\mathrm{n}^{0}$ 50, p. 189-208.

QuÉva C., 2015. De l'espace à faible densité à l'espace diffus : essai de définition, in Devès C. (dir.), Vivre et travailler dans les espaces à faible densité. Quelles stratégies de développement?, Paris, L'Harmattan, p. 17-33.

Quéva C., Brès A., 2016. Les espaces de faible densité, entre aménagement et développement local, in Desjardins, X., Géneau de Lamarlière I. (dir.), L'aménagement du territoire en France, Paris, La Documentation française, p. 143159.

Torre A., Filippi M. (dir.), 2005. Proximités et changements sociodémographiques dans les mondes ruraux, Paris, INRA éditions, $322 \mathrm{p}$.

Torre A., 2009. Retour sur la notion de proximité géographique, Géographie, Économie et Société, vol. 11, n 1, p. 63-75.

Williamson O., 1994, Les institutions de l'économie, Paris, InterÉditions, $404 \mathrm{p}$.

Wolman H., Page E., 2002. Policy Transfer among Local Governments: An Information-Theory Approach, Governance, vol. 15, no 4, p. 477-501. 